\title{
Expressão imuno-histoquímica das metaloproteinases \\ 2 e 9 não está associada à progressão do carcinoma de células escamosas de esôfago
}

\author{
Metalloproteinases 2 and 9 immunohistochemistry expression is not associated to esophageal squamous cell \\ carcinoma progression
}

Izabella Paz Danezi Felin'; Ivana Grivicich²; Carlos Roberto Felin³; Carlos Alexandre Fedrigo4; Adriana Brondani da Rocha ${ }^{5}$

\section{unitermos}

Carcinoma de células escamosas de esôfago

Metaloproteinase 2

Metaloproteinase 9

Imuno-histoquímica

\section{resumo}

Introdução: O carcinoma de células escamosas do esôfago está entre os tipos mais agressivos de câncer e de pior prognóstico. As metaloproteinases de matriz (MMPs), especialmente as MMP-2 e MMP-9, vêm sendo utilizadas para avaliação prognóstica do câncer, associadas a invasão, tamanho e crescimento tumoral. Objetivo: O presente estudo visa investigar as expressões imuno-histoquímicas de MMP-2 e MMP-9, avaliando se existe correlação entre sua expressão e o estadiamento tumoral, invasão vascular, invasão local (pT) e diferenciação tumoral no carcinoma de células escamosas de esôfago. Material e Método: Foi realizado um estudo retrospectivo utilizando 31 blocos de parafina contendo tumores de carcinoma escamoso esofágico, obtidas por esofagectomias realizadas entre 1998 e 2003, no Hospital Universitário de Santa Maria (HUSM). Os cortes histológicos foram submetidos à reação imuno-histoquímica, com sistema de amplificação por polímero não-biotinilado Novolink para deteç̧ão de MMP-2 e MMP-9. Resultados: A avaliação da MMP-2 apresentou positividade fraca em apenas cinco casos, não demonstrando correlação com as variáveis estudadas. Também não foram observadas associações significativas entre as variáveis do estudo e o grau de expressão imuno-histoquímica da MMP-9. Conclusão: A expressão imuno-histoquímica das MMP-2 e MMP-9 não parece ser influenciada pelos parâmetros investigados. Nesse sentido, estudos adicionais são necessários para melhor compreensão de sua associação aos fatores prognósticos do carcinoma de células escamosas de esôfago. abstract

Introduction: Esophageal squamous cell carcinoma is an aggressive malignant neoplasia with poor prognosis. The expression of matrix metalloproteinases (MMPs), mainly 2 and 9, has been used for the prognostic evaluation of cancer in association with tumor invasion, size and tumoral growth analysis. Objective: The aim of the present study was to investigate the immunohistochemical expression of MMP-2 and MMP-9 and evaluate if there is an association between their expression and tumor staging, vascular invasion, local invasion ( $P T)$ and tumoral differentiation in esophageal squamous cell carcinoma. Material and Methods: We conducted a retrospective study using 31 paraffin-embedded specimens of esophageal squamous cell carcinoma obtained by esophagectomies performed at Santa Maria University Hospital, Rio Grande do Sul State, Brazil between 1998 and 2003. The histological sections were immunohistochemically studied using a non-biotinylated Novolink system for MMP-2 and MMP-9 detection. Results: MMP-2 immunohistochemical expression was detected only in 5 cases and did not demonstrate correlation with the variables analyzed. Furthermore, MMP-9 immunohistochemical expression was not significantly associated with the other variables. Conclusion: MMP-2 and MMP-9 immunohistochemical expression does not seem to be influenced by the variables reported in this study. Thus further investigations are required to a better understanding of their association with the prognostic factors of esophageal squamous cell carcinoma.

\section{key words}

Esophageal squamous cell carcinoma

Metalloproteinase 2

Metalloproteinase 9

Immunohistochemistry

\footnotetext{
1. Médica patologista; professora do Departamento de Patologia da Universidade Federal de Santa Maria (UFSM); doutoranda do Programa de Pós-Graduação em Cenética e Toxicologia Aplicada, da Universidade Luterana do Brasil (ULBRA).

2. Bióloga; doutora em Cenética e Biologia Molecular pela Universidade Federal do Rio Grande do Sul (UFRCS); professora do Programa de Pós-Graduação em Cenética e Toxicologia Aplicada e do Programa de Pós-Graduação em Diagnóstico Genético e Molecular da ULBRA; pesquisadora do Laboratório de Marcadores de Estresse Celular do Centro de Pesquisas em Ciências Médicas (CPCM) da ULBRA.

3. Médico oncologista do Hospital da Brigada Militar de Santa Maria (HBM/SM) e do Hospital Universitário (HU) da UFSM; doutorando do Programa de Pós-Graduação em Cenética e Toxicologia Aplicada da ULBRA.

4. Biólogo; mestrando do Programa de Pós-Graduação em Cenética e Toxicologia Aplicada da ULBRA; pesquisador do Laboratório de Marcadores de Estresse Celular do CPCM/ULBRA.

5. Bióloga, doutora em Bioquímica pela UFCRS; professora do Programa de Pós-Graduação em Cenética e Toxicologia Aplicada e do Programa de Pós-Graduação em Diagnóstico Genético e

Molecular da ULBRA; pesquisadora do Laboratório de Marcadores de Estresse Celular do CPCM/ULBRA.
} 


\section{Introdução}

O câncer de esôfago é uma doença agressiva que representa cerca de $2 \%$ de todos os tumores malignos e a terceira causa mais comum de câncer do trato gastrointestinal ${ }^{(16,34)}$. O carcinoma de células escamosas é o tumor de esôfago mais frequente com aproximadamente 10.600 casos novos por ano no mundo ${ }^{(19)}$. Geralmente está associado a um prognóstico ruim, por se tratar de um tumor em estádio avançado no momento do diagnóstico e apresentar alta frequência de metástases linfonodais ${ }^{(10,34)}$.

A invasão local e a capacidade de metastatização são considerados importantes critérios que conferem a uma neoplasia a característica de malignidade ${ }^{(3,5,28)}$. Assim, a invasão de um tumor epitelial é uma etapa essencial para a progressão do câncer, incluindo os tumores de esôfago ${ }^{(6)}$. Nesse sentido, a degradação dos componentes da matriz extracelular confere ao tumor de esôfago a possibilidade de invasão local para tecidos adjacentes, além de permitir o acesso aos vasos sanguíneos e linfáticos, proporcionando a disseminação a distância $(5,32,36)$. A degradação dos componentes da matriz extracelular é mediada por uma família de enzimas proteolíticas, denominadas metaloproteinases de matriz (MMPs). As MMP-2 e MMP-9 são gelatinases, zinco-dependentes, com função na degradação da matriz extracelular, incluindo a membrana basal ${ }^{(4,20,29)}$. Dessa forma, as MMP-2 e MMP-9 apresentam importante papel na angiogênese, progressão tumoral e disseminação metastática do câncer $^{(2,8,29,36)}$. A expressão elevada de MMPs, incluindo as MMP-2 e MMP-9, tem sido observada em vários tipos de tumores malignos ${ }^{(4,33,36,38)}$. Por outro lado, está bem estabelecido que os níveis de expressão das MMP-2 e MMP-9 dependem do contexto biológico, como por exemplo, a presença de regiões promotoras bastante complexas $^{(9)}$.

A associação entre as MMP-2 e MMP-9 com a tumorigênese do carcinoma de células escamosas de esôfago tem sido relatada em alguns estudos ${ }^{(21,27,37)}$. Entretanto, a associação da expressão das MMP-2 e MMP-9 em câncer de esôfago aos parâmetros histológicos ainda deve ser elucidada. Neste estudo, avaliamos as expressões imuno-histoquímicas de MMP-2 e MMP-9 em amostras de carcinoma de células escamosas de esôfago e sua correlação com estadiamento, invasão local, invasão vascular e grau de diferenciação tumoral.

\section{Material e método}

\section{Seleção de casos}

Foram selecionados 31 blocos de parafina contendo produtos de ressecção cirúrgica por esofagectomia, diagnosticados como carcinoma de células escamosas de esôfago humano. Os blocos foram obtidos do arquivo do Serviço de Patologia do Hospital Universitário de Santa Maria (HUSM), referentes aos exames histopatológicos rotineiros no período de 1998 a 2003.

Como critérios de inclusão, foram consideradas a existência e a conservação de todos os blocos de parafina referentes a cada caso no período de tempo destinado ao estudo. Como critérios de exclusão, foram utilizados má conservação dos blocos de parafina, arquivo incompleto, dúvidas diagnósticas, outros diagnósticos, produtos de biópsias, casos fora do período estipulado, casos em que os pacientes tenham sido submetidos à quimioterapia e ou radioterapia neoadjuvante e casos com artefatos durante fixação e processamento.

O protocolo experimental, desenvolvido neste estudo, foi submetido e aprovado pelo Comitê de Ética em Pesquisa em Seres Humanos e Animais da ULBRA (protocolo CEPULBRA 2006-266H).

\section{Processamento histológico}

Foram obtidos cortes histológicos de $3 \mu \mathrm{m}$ de espessura dos blocos de parafina, montados em lâminas de vidro tratadas com organosilano (3-aminopropiltrietoxi-silano; Sigma Chemical $\mathrm{Co}^{\circledast}$, St. Louis, MO, USA) e coradas com hematoxilina e eosina para reavaliação histopatológica. A revisão histopatológica foi realizada por dois patologistas experientes, de forma independente, sem identificação dos casos, na forma de duplo cego. Eventuais resultados conflitantes foram discutidos pelos mesmos para definição consensual da análise.

Procedeu-se graduação, estadiamento pTNM e caracterização de itens relativos ao preenchimento de um instrumento recomendado para câncer esofágico ${ }^{(17)}$ e adotado pelo Serviço de Patologia do HUSM. Dos itens envolvidos na padronização do laudo anatomopatológico em esôfago, foram selecionados os mais relacionados com o prognóstico em câncer de esôfago. Neste estudo avaliamos estadiamento do tumor (sistema pTNM), invasão vascular, invasão local (pT do pTNM), e grau de diferenciação tumoral. 


\section{Análise imuno-histoquímica}

Os espécimes emblocados em parafina foram submetidos a cortes de $3 \mu \mathrm{m}$ de espessura e montados em lâminas de vidro previamente preparadas com organosilano (3-aminopropiltrietoxi-silano; Sigma Chemical $\mathrm{Co}^{\circledR}$, St. Louis, MO, USA) para a realização da técnica imuno-histoquímica. A atividade de peroxidase endógena foi bloqueada com solução de peróxido de hidrogênio a $3 \%$ em solução tampão salina de fosfato (PBS) por 10 minutos e a recuperação antigênica foi realizada em vaporizador por 60 minutos, utilizando tampão de ácido cítrico a $0,21 \% \mathrm{em} \mathrm{pH} 6$. Os cortes foram posteriormente incubados com os anticorpos primários anti-MMP-2 (1:400, clone 17B11) e anti-MMP-9 (1:400, clone 15W2), ambos da Novocastra ${ }^{\circledR}$, New Castle Upon Tyne, UK, durante $12 \mathrm{~h}$ à temperatura ambiente. Após a incubação, foi aplicado o sistema de amplificação por polímero não biotinilado Novolink (Novocastra ${ }^{\circledR}$, New Castle Upon Tyne, UK) por 60 minutos à temperatura ambiente. Para a revelação das reações, foi utilizada a diaminobenzidina (DAB; Sigma Chemical $\mathrm{Co}^{\circledR}$, St. Louis, MO, USA) e contra coloração com hematoxilina de Harris.

Como controles positivos para MMP-2, foram utilizadas células deciduais normais e, para MMP-9, células de fígado humano normal. O controle negativo foi feito pela omissão do anticorpo primário.

As lâminas foram analisadas em microscópio óptico Olympus CX31, estimando-se o percentual de células positivas de forma semiquantitativa em menor aumento (40x). Foi analisado o percentual de células neoplásicas coradas, considerando apenas expressão citoplasmática nas células tumorais. Os casos foram graduados conforme o seguinte critério: 0 - negativa: $<5 \%$ de células; 1 - positividade fraca: $5 \%$ a $20 \%$ das células; 2 - positividade moderada: $21 \%$ a $50 \%$ das células; 3 - positividade forte: $>50 \%{ }^{(32)}$.

\section{Análise estatística}

Para verificar a associação entre a MMP-9 e as variáveis invasão local, invasão vascular, estadiamento tumoral e grau de diferenciação do tumor, foi usado o teste exato de Fisher. Para aplicar o teste, os níveis $0+1$ e $2+3$ referentes à MMP-9 foram agrupados. Além disso, foram considerados na análise somente os extremos de cada variável. Para a variável diferenciação tumoral, foi usado pobremente diferenciado e bem diferenciado, para invasão local foi usado pT2 e pT3, pois pT1 e pT4 tinham baixas frequências; para estadiamento foram usados IIA e III, pois o estádio I apresentou baixa frequência e para invasão vascular foi considerado os critérios positivo e negativo. Foi adotado o nível de significância de 0,05 e os cálculos foram executados com o programa Statistical Package of Social Science (SPSS), versão 14.0.

A verificação de associação entre MMP-2 com as variáveis diferenciação, invasão local e estadiamento não foi realizada devido à baixa frequência de expressão desse marcador (cinco casos, todos nível 1).

\section{Resultados}

\section{Dados da amostra}

Neste estudo foram analisados 31 casos de carcinoma de células escamosas de esôfago, tendo um predomínio do sexo masculino (25 casos; $80,6 \%$ ), com idade média de 56 anos. Em relação à localização, a maioria dos tumores, 16 casos $(51,6 \%)$, foram encontrados no terço inferior do esôfago, 13 casos $(41,9 \%)$ no terço médio, um caso $(3,2 \%)$ no terço superior e um caso $(3,2 \%)$ nos terços médio e inferior concomitantemente (Tabela 1).

A avaliação da diferenciação tumoral revelou 14 casos $(45,2 \%)$ moderadamente diferenciados, 12 (38,7\%) bem diferenciados e cinco $(16,1 \%)$ pobremente diferenciados. Em 25 casos $(80,6 \%)$, não foi detectada invasão vascular (Tabela 1). De acordo com o estadiamento tumoral TNM (T: tumor, N: linfonodo, M: metástase), 13 casos $(41,9 \%)$ foram classificados como estádio IIA, $13(41,9 \%)$ foram estádio III, três $(9,7 \%)$, estádio IIB e dois $(6,4 \%)$, estádio I. Dessa forma, os resultados apontam uma predominância para os estágios II A e III (Tabela1).

\section{Análise imuno-histoquímica de MMP-2 e MMP-9}

A expressão da MMP-2 só foi detectada em cinco casos, todos com expressão fraca do anticorpo (nível 1) 


\begin{tabular}{|c|c|c|}
\hline \multirow{2}{*}{$\begin{array}{l}\text { Tabela } 1 \\
\text { Característica }\end{array}$} & \multicolumn{2}{|c|}{$\begin{array}{l}\text { Características gerais das amostras } \\
\text { de carcinoma de células escamosas } \\
\text { de esôfago }\end{array}$} \\
\hline & & $\mathrm{N}^{0}$ casos \\
\hline \multirow{3}{*}{ Sexo } & Masculino & 25 \\
\hline & Feminino & 6 \\
\hline & $<50$ & 11 \\
\hline \multirow{3}{*}{ Idade (anos) } & $51-60$ & 10 \\
\hline & $61-70$ & 7 \\
\hline & $>70$ & 3 \\
\hline \multirow{4}{*}{$\begin{array}{l}\text { Localização } \\
\text { do tumor }\end{array}$} & Terço superior & 1 \\
\hline & Terço médio & 13 \\
\hline & Terço inferior & 16 \\
\hline & $\begin{array}{l}\text { Terço médio e } \\
\text { inferior }\end{array}$ & 1 \\
\hline \multirow{3}{*}{$\begin{array}{l}\text { Grau de } \\
\text { diferenciação }\end{array}$} & Bem diferenciado & 12 \\
\hline & $\begin{array}{l}\text { Moderadamente } \\
\text { diferenciado }\end{array}$ & 14 \\
\hline & $\begin{array}{l}\text { Pobremente } \\
\text { diferenciado }\end{array}$ & 5 \\
\hline \multirow{2}{*}{$\begin{array}{l}\text { Invasão } \\
\text { vascular }\end{array}$} & Positiva & 6 \\
\hline & Negativa & 25 \\
\hline \multirow{2}{*}{$\begin{array}{l}\text { Invasão } \\
\text { perineural }\end{array}$} & Positiva & 8 \\
\hline & Negativa & 23 \\
\hline \multirow{4}{*}{ Estadiamento } & I & 2 \\
\hline & $\| A$ & 13 \\
\hline & $\| \mathrm{B}$ & 3 \\
\hline & III & 13 \\
\hline
\end{tabular}

(Figura A). Já a presença da MMP-9 pode ser claramente visualizada no citoplasma das células (Figura B).

Dos 31 casos estudados, $25(80,6 \%)$ apresentaram invasão vascular negativa e seis casos $(19,3 \%)$, invasão vascular positiva. Entre os casos de invasão vascular negativa, 20 (64,5\%) apresentaram expressão da MMP-9 negativa ou fraca (0 ou 1) e em 5 casos (16,1\%), a expressão da MMP-9 foi moderada ou forte (2 ou 3). Para os casos de invasão vascular positiva, quatro (12,9\%) demonstraram MMP-9 negativa ou fraca (0 ou 1$)$ e 2 casos $(6,4 \%)$ foram MMP-9 moderada ou forte (2 ou 3) (Tabela 2). Em relação à MMP-2, dos cinco casos de marcação fraca, quatro (80\%) foram invasão vascular negativa e um (20\%), invasão vascular positiva. Devido ao baixo número de casos com imunomarcação para MMP-2, não realizamos a correlação desse marcador com os demais parâmetros.

Para os demais critérios estudados (diferenciação, invasão local e estadiamento), foram utilizados os dois extremos de cada variável analisada. Assim, de um total de 31 casos estudados, foi considerado o número de casos de cada extremo em relação a cada critério. Considerando o número de casos de cada extremo, em relação à diferenciação tumoral, foram 17 casos estudados. Destes, cinco $(29,4 \%)$ foram classificados como pobremente diferenciados e 12 (70,6\%), como bem diferenciados. Entre os casos pobremente diferenciados, quatro $(23,5 \%)$ apresentaram MMP-9 (0 ou 1) e um caso (5,9\%), MMP-9 (2 ou 3). Enquanto isso, nos casos classificados como bem diferenciados, foi observada uma expressão da MMP-9 (0 ou 1) em oito casos (47\%) e MMP-9 (2 ou 3) em quatro (23,5\%) (Tabela 3).

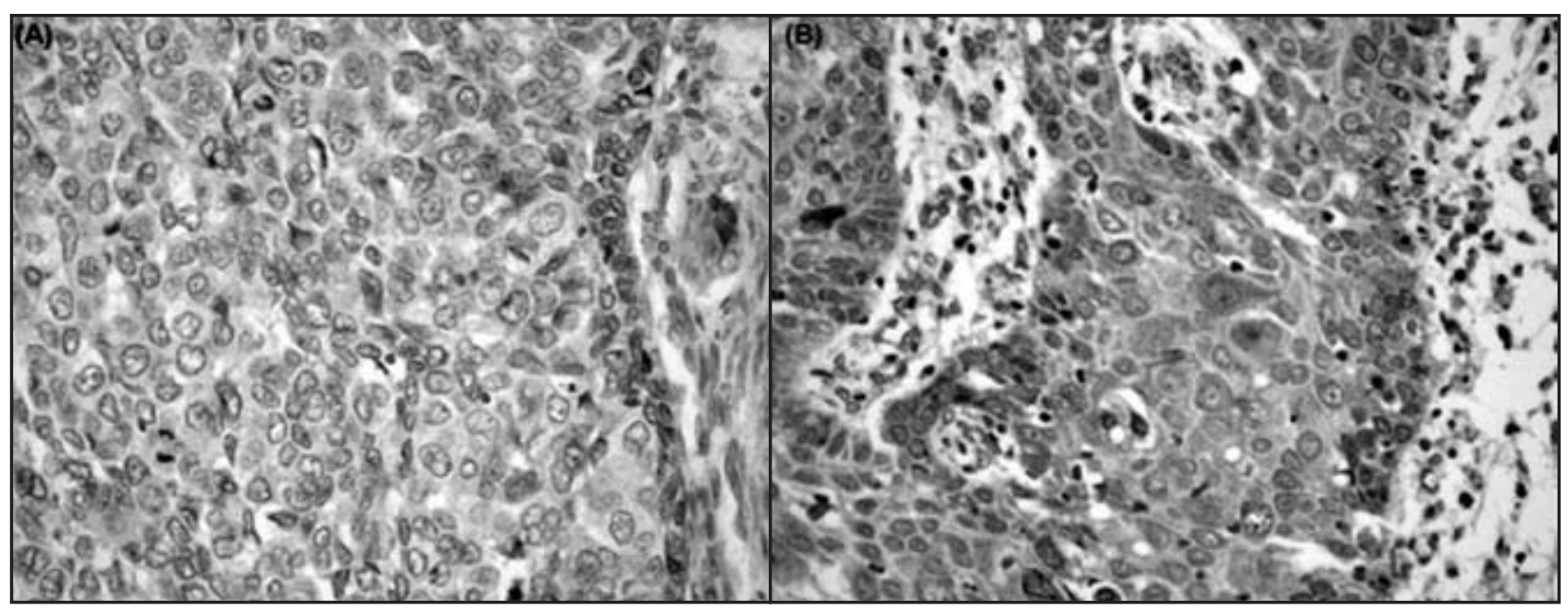

Figura - Marcação imuno-histoquímica pela MMP-2 (A) e MMP-9 (B) em carcinoma de células escamosas de esôfago (400x) MMP: metaloproteinase de matriz. 


$\begin{array}{lccc}\text { Tabela 2 } & \begin{array}{l}\text { Associação entre MMP-9 e invasão } \\ \text { vascular }\end{array} & & \\ & \begin{array}{c}\text { Invasão } \\ \text { vascular } \\ \text { negativa }\end{array} & \begin{array}{c}\text { Invasão } \\ \text { vascular } \\ \text { positiva }\end{array} & \text { Total } \\ & 20 & 4 & 24 \\ \text { MMP-9 } & (64,5 \%) & (12,9 \%) & (77,4 \%) \\ \text { (0 ou 1) } & 5 & 2 & 7 \\ \text { MMP-9 } & (16,1 \%) & (6,4 \%) & (22,6 \%) \\ \text { (2 ou 3) } & 25 & 6 & 31 \\ \text { Total } & (80,6 \%) & (19,3 \%) & (100 \%)\end{array}$

MMP-9: metaloproteinase 9 .

\section{Associação entre MMP-9 e}

Tabela 3 diferenciação tumoral

\begin{tabular}{lccc}
\hline & $\begin{array}{r}\text { Pobremente } \\
\text { diferenciado }\end{array}$ & $\begin{array}{c}\text { Bem } \\
\text { diferenciado }\end{array}$ & Total \\
MMP-9 & 4 & 8 & 12 \\
(0 ou 1) & $(23,5 \%)$ & $(47 \%)$ & $(70,68 \%)$ \\
MMP-9 & 1 & 4 & 5 \\
(2 ou 3) & $(5,9 \%)$ & $(23,5 \%)$ & $(29,4 \%)$ \\
Total & 5 & 12 & 17 \\
& $(29,4 \%)$ & $(70,6 \%)$ & $(100 \%)$
\end{tabular}

MMP-9: metaloproteinase 9.

Quanto à invasão local, foram analisados 28 casos (Tabela 4). Dos oito casos pT2 (21,4\%), seis apresentaram MMP-9 (0 ou 1) e dois (7,1\%), MMP-9 (2 ou 3). Enquanto isso, entre os casos pT3, $15(53,6 \%)$ foram MMP-9 (0 ou 1) e cinco (17,8\%), MMP-9 (2 ou 3) (Tabela 4). Nesse critério, a maioria dos casos estudados (75\%) foi MMP-9 (0 ou 1) e estágios pT3 (71,4\%) (Tabela 5).

Quando analisamos o estadiamento do tumor, 26 casos foram utilizados. Confrontando MMP-9 com estadiamento do tumor, 11 (42,3\%) apresentaram estádio IIA e MMP-9 (0 ou 1); dois (7,7\%), estádio IIA e MMP-9 (2 ou 3); nove (34,6\%), estádio III e MMP-9 (0 ou 1$)$ e quatro $(15,4 \%)$, estádio III e MMP-9 (2 ou 3) (Tabela 4).

\section{Discussão}

Os pacientes portadores de carcinoma escamoso de esôfago apresentam um prognóstico reservado, mesmo quando submetidos à ressecção cirúrgica, dado o caráter infiltrativo dessas neoplasias ${ }^{(34)}$. A migração e a invasão

\section{Associação entre MMP-9 e}

Tabela 4 estadiamento tumoral

Estadiamento Estadiamento II A

III

Total

$\begin{array}{llll}\text { MMP-9 } & 11 & 9 & 20 \\ \text { (0 ou 1) } & (42,3 \%) & (34,6 \%) & (76,9 \%) \\ \text { MMP-9 } & 2 & 4 & 6 \\ \text { (2 ou 3) } & (7,7 \%) & (15,4 \%) & (23,1 \%) \\ \text { Total } & 13 & 13 & 26 \\ & (50 \%) & (50 \%) & (100 \%)\end{array}$

MMP-9: metaloproteinase 9.

\section{Tabela 5 Associação entre MMP-9 e invasão local (pT)}

$\begin{array}{lccc} & \text { Invasão local } & \text { Invasão local } & \text { Total } \\ & \text { pT2 } & \text { pT3 } & \\ \text { MMP-9 } & 6 & 15 & 21 \\ \text { (0 ou 1) } & (21,4 \%) & (53,6 \%) & (75 \%) \\ \text { MMP-9 } & 2 & 5 & 7 \\ \text { (2 ou 3) } & (7,1 \%) & (17,8 \%) & (25 \%) \\ & 8 & 20 & 28 \\ \text { Total } & (28,6 \%) & (71,4 \%) & (100 \%)\end{array}$

MMP-9: metaloproteinase 9.

dos tumores malignos representam eventos essenciais na progressão neoplásica. A degradação da membrana basal e a migração de células malignas no estroma é um complexo processo que requer interações sequenciais entre células tumorais, matriz extracelular, células inflamatórias e estromais( ${ }^{(2)}$. O estudo da expressão de MMPs em tumores humanos tem demonstrado que essas proteinases são um importante componente do fenótipo invasivo em várias neoplasias, incluindo tumores do trato gastrointestinal $(4,12,14)$. Nesse sentido, a expressão desregulada de MMP-2 e MMP-9 desempenha um papel crítico na invasão da membrana basal por meio da degradação do colágeno tipo IV e, portanto, na iniciação, vascularização e progressão de diversos tumores ${ }^{(25)}$.

Mais recentemente, foi relatado que a MMP-9 exerce um papel mais pronunciado do que a MMP-2 durante a invasão $0^{(7)}$. Nesse aspecto, nosso estudo demonstrou uma maior variabilidade de expressão da MMP-9 nos casos avaliados, enquanto a expressão da MMP-2 foi negativa para a maioria dos casos e fraca em apenas cinco casos. Ainda que alguns estudos tenham encontrado associação entre a 
hiperexpressão de MMP-2 com o avanço do estadiamento e o grau de tumores esofágicos ${ }^{(13,37)}$, nossa amostra não apresentou tal associação. Entretanto, diversos estudos mostraram que o aumento da expressão das MMP-2 e MMP-9 em território invasivo se dá pelo aumento de secreção de MMPs pelas células estromais de tumores de esôfago ${ }^{(15,27,30,31)}$. Nesse sentido, vários achados experimentais que demonstram associação entre tumorigênese e progressão tumoral com a expressão das MMPs são oriundos de estudos que contemplam a resposta adjacente inflamatória e tecido conjuntivo às células neoplásicas (7). Entre estes, Samantaray, et al. demonstraram um aumento de RNA mensageiro (RNAm) da MMP-2, especialmente em componente estromal de tumor de esôfago ${ }^{(27)}$. Assim, a baixa expressão da MMP-2 observada no nosso estudo pode ser explicada pelo fato de ter sido avaliada somente no citoplasma das células neoplásicas, sem considerarmos as células estromais.

Existem evidências de que maiores expressão e ativação da MMP-9 são dependentes da densidade das células tumorais, bem como da localização intratumoral (centro ou periferia do tumor) onde foi avaliada a expressão destas proteinases ${ }^{(11)}$. Os estudos que quantificam esse fator levam em conta apenas a expressão na borda do tumor ${ }^{23,}{ }^{24)}$. No nosso estudo, foi verificada a expressão citoplasmática intratumoral nas células neoplásicas, o que pode ter determinado a ausência de associação com os critérios anatomopatológicos selecionados.

Apesar da associação entre MMP-2 e MMP-9 com angiogênese, progressão tumoral e metástases ${ }^{(2,8,29,36)}$, alguns estudos não apresentaram essa correlação. Väisänen, et al. compararam o valor prognóstico das MMP-2 e MMP-9 em melanoma cutâneo, não detectando relevância prognóstica na imunorreatividade da MMP-9 em melanoma primário em células tumorais e estromais ${ }^{(35)}$. Masuda e Aoki demonstraram que não existe diferença significativa entre a expressão do RNAm da MMP-9 e da MMP-2 entre células tumorais de cólon e mucosa saudável(18). Também em câncer de cólon a expressão das MMP-2 e MMP-9 não demonstrou correlação com o estádio tumoral(26). Recentemente, foi demonstrado que a MMP-2 não parece contribuir com a carcinogênese no câncer de esôfago(1).

Além disso, apesar de a expressão da MMP-2 ser constitutiva, a atividade do promotor dessa protease pode ser induzida. Essa regulação depende da ativação de vários fatores de transcrição que podem ativar ou inativar a região do promotor da MMP-2 ${ }^{(22)}$. Mais ainda, o aumento da expressão das MMP-2 e MMP-9 parece estar mais associado a lesões iniciais do que a carcinomas de esôfago já estabelecidos. A hiperexpressão de MMP-2 e MMP-9 tem sido identificada como parte de um evento inicial na tumorigênese, associado à degradação da membrana basal e à transformação maligna, mas não necessariamente ao potencial de malignidade ${ }^{(21,27,37)}$. Dessa forma, podemos sugerir que o aumento da expressão das MMP-2 e MMP-9 em território invasivo de carcinoma de esôfago deve estar mais associado ao incremento de secreção das MMPs pelas células estromais e inflamatórias, ou ainda depender da ativação da região promotora dessa protease.

\section{Referências}

1. AUGOFF, K. et al. Expression of decorin in esophageal cancer in relation to the expression of three isoforms of transforming growth factor-beta (TGF-beta1, -beta2, and -beta3) and matrix metalloproteinase-2 activity. Cancer Invest, v. 27, p. 443-52, 2009.

2. BERGERS, G. et al. Matrix metalloproteinase- 9 triggers the angiogenic switch during carcinogenesis. Nat Cell Biol, v. 2, p. 737-44, 2000.

3. BJÖRKLUND, M.; KOIVUNEN, E. Gelatinase-mediated migration and invasion of cancer cells. Biochim Biophys Acta, v. 1755, n. 1, p. 37-69, 2005.

4. CLARK, I.M. et al. The regulation of matrix metalloproteinases and their inhibitors. Int J Biochem Cell Biol, v. 40, p. 1362-78, 2008.

5. COTRAN, R.S.; KUMAR, V. Robbins Patologia estrutural e funcional. Rio de Janeiro: Guanabara Koogan, 2006.

6. D’AMICO, T.A. Molecular biologic staging of esophageal cancer. Thorac Surg Clin, v. 16, n. 4, p. 317-27, 2006.
7. ERDEM, N.F. et al. Characterization of 3 oral squamous cell carcinoma cell lines with different invasion and/or metastatic potentials. J Oral Maxillofac Surg, v. 65, p. 1725-33, 2007.

8. HESLIN, M.J. et al. Role of matrix metalloproteinases in colorectal carcinogenesis. Annals of Surgery, v. 233, n. 6, p. 786-92, 2001.

9. HU, J. et al. Matrix metalloproteinase inhibitors as therapy for inflammatory and vascular diseases. Nature, v. 6, p. 480-8, 2007.

10. IKEDA, G. et al. Prognostic significance of biologic factors in squamous cell carcinoma of the esophagus. Cancer, v. 86, n. 8, p. 1396-405, 1999.

11. ILLEMANN, M. et al. MMP-9 is differentially expressed in primary human colorectal adenocarcinomas and their metastases. Mol Cancer Res, v. 4, p. 293-302, 2006.

12. IMAMURA, T. et al. Expression of membranetype metalloproteinase- 1 in human pancreatic 
adenocarcinomas. J Cancer Res Clin Oncol, v. 124, p. 65-72, 1998.

13. ISHIBASHI, Y. et al. CD147 and matrix metalloproteinase-2 protein expression as significant prognostic factors in esophageal squamous cell carcinoma. Cancer, v. 101, n. 9, p. 1994-2000, 2004.

14. KJELLMAN, M. et al. Gelatinase A and membrane-type 1 matrix metalloproteinase mRNA: expressed in adrenocortical cancers but not in adenomas. World $\mathrm{J}$ Surg, v. 23, p. 237-42, 1999.

15. KOYAMA, H. et al. Gelatinolytic activity of matrix metalloproteinase-2 and 9 in esophageal carcinoma; a study using in situ zymography. Eur J Cancer, v. 36, p. 2164-70, 2000.

16. KUWANO, H. et al. Distinctive clinicopathological characteristics in esophageal squamous cell carcinoma. Ann Thorac Cardiovasc Surg, v. 9, n. 1, p. 6-13, 2003.

17. LEE, R.G.; COMPTON, C.C. Protocol for the examination of specimens removed from patients with esophageal carcinoma. A basis for checklists. The Cancer Committee, College of American Pathologists, and the Task Force on the Examination of Specimens From Patients With Esophageal Cancer. Arch Pathol Lab Med, v. 121, p. 925-9, 1997.

18. MASUDA, H.; AOKI, H. Host expression of matrix metalloproteinase-2 and tissue inhibitor of metalloproteinase-2 in normal colon tissue affects metastatic potential of colorectal cancer. Dis Colon Rectum, v. 42, p. 393-7, 1999.

19. MINISTÉRIO DA SAÚDE. Estimativa 2005: incidência de câncer no Brasil. Rio de Janeiro: INCA, 2005.

20. NAGASE, G.; WOESSNER Jr., F. Matrix metalloproteinases. J Biol Chem, v. 274, n. 31, p. 21491-4, 1999.

21. OHASHI, K. et al. Increased expression of matrix metalloproteinase 7 and 9 and membrane type 1matrix metalloproteinase in esophageal squamous cell carcinomas. Cancer, v. 88, p. 2201-09, 2000.

22. OLAF, R.F. et al. The role of gelatinases in colorectal cancer progression and metastasis. Biochimica et Biophysica Acta, v. 1705, p. 69- 89, 2004.

23. PAPADOPOULOU, S. et al. Expression of gelatinase-A (MMP-2) in human colon cancer and normal colon mucosa. Tumour Biol, v. 22, p. 383-9, 2001.

24. PYKE, C. et al. Messenger RNA for two type IV collagenases is located in stromal cells in human colon cancer. Am J Pathol, v. 142, p. 359-65, 1993.

25. RAO, J.S. et al. MMP-9 SiRNA induced senescence resulting in inhibition of medulloblastoma growth via p16INK4A and MAPK pathway. Cancer Res, v. 67, n. 10, p. 4956-64, 2007.
26. RING, P. et al. Expression of tissue inhibitor of metalloproteinases TIMP-2 in human colorectal cancera predictor of tumour stage. $\mathrm{Br} J$ Cancer, v. 76, p. 805-11, 1997.

27. SAMANTARAY, S. et al. Increased expression of MMP-2 and MMP-9 in esophageal squamous cell carcinoma. $J$ Cancer Res Clin Oncol, v. 130, p. 37-44, 2004.

28. SCHÄFER, M.; WERNER, S. Cancer as an overhealing wound: an old hypothesis revisited. Nat Rev Mol Cell Biol, v. 9, p. 628-38, 2008.

29. SCHNALFELDT, B. et al. Increased expression of matrix metalloproteinases (MMP)-2, (MMP)-9, and the urokinase-type plasminogen activator is associated with progression from benign to advanced ovarian cancer. Clinical Cancer Research, v. 7, p. 2396404, 2001.

30. SHIMA, I. et al. Production of matrix metalloproteinase-2 and metalloproteinase- 3 related to malignant behavior of esophageal carcinoma. A clinicopathologic study. Cancer, v. 70, p. 2747-53, 1992.

31. SHIMA, l. et al. Production of matrix metalloproteinase 9 (92-kDa gelatinase) by human oesophageal squamous cell carcinoma in response to epidermal growth factor. Br J Cancer, v. 67, n. 4, p. 721-7, 1993.

32. SOARES, F.A. et al. The expression of metaloproteinases- 2 and -9 is different according to the patterns of growth and invasion in squamous cell carcinoma of the penis. Virchows Arch, v. 449, n. 6, p. 637-46, 2006.

33. TURPEENNIEMI-HUJANEN, T. Gelatinases (MMP-2 and9) and their natural inhibitors as prognostic indicators in solid cancers. Biochimie, v. 87, p. 287-97, 2005.

34. UMAR, S.B.; FLEISCHER, D.E. Esophageal cancer: epidemiology, pathogenesis and prevention. Nat Clin Pract Gastroenterol Hepatol, 2008.

35. VÄISÄNEN, A.H.; KALLIOINEN, M.; TURPEENNIEMIHUJANEN, T. Comparison of the prognostic value of matrix metalloproteinases 2 and 9 in cutaneous melanoma. Hum Pathol, v. 39, n. 3, p. 377-85, 2008.

36. WESTERMARCK, J.; KÄHÄRI, V-M. Regulation of matrix metalloproteinase expression in tumor invasion. The FASEB J, v. 13, n. 8, p. 781-92, 1999.

37. YAMAMOTO, H. et al. Association of matrilysin expression with recurrence and poor prognosis in human esophageal squamous cell carcinoma. Cancer Res, v. 59, p. 3313-6, 1999.

38. YOSHIZAKI, T.; SATO, H.; FURUKAWA, M. Recent advances in the regulation of matrix metalloproteinase 2 activation: from basic research to clinical implication. Oncol Rep, v. 9, n. 3, p. 607-11, 2002.

\begin{tabular}{l|l} 
& Endereço para correspondência \\
\hline Adriana Brondani da Rocha \\
Laboratório de Marcadores de Estresse Celular do CPCM \\
da ULBRA. \\
Av Farroupilha, 8001, Prédio 22, $5^{\circ}$ andar, Canoas - RS. \\
CEP: $92245-900$ \\
Tel: $+55-51-34779219$ \\
e-mail: brondani@terra.com.br
\end{tabular}

